\title{
Sequence and expression analysis of histone deacetylases in rice
}

\author{
Wenqun $\mathrm{Fu}^{\mathrm{a}, \mathrm{c}}$, Keqiang $\mathrm{Wu}^{\mathrm{b}, *}$, Jun Duan ${ }^{\mathrm{a}, *}$ \\ ${ }^{a}$ South China Botanical Garden, Chinese Academy of Sciences, Guangzhou 510650, China \\ ${ }^{\mathrm{b}}$ Institute of Plant Biology, National Taiwan University, Taipei 106, Taiwan \\ ${ }^{\mathrm{c}}$ Graduate School of the Chinese Academy of Sciences, Beijing 100039, China
}

Received 28 February 2007

Available online 12 March 2007

\begin{abstract}
Histone acetylation levels are determined by the action of histone acetyltransferases and histone deacetylases (HDACs). Sequence similarity and profile searching tools were used to analyze the genome sequence of rice (Oryzae sativa) for genes encoding HDAC proteins. The rice RPD3/HDA1-family HDAC proteins can be divided into four classes based on sequence similarity and phylogenetic analysis of sequences obtained from the rice genome. The spatial expression pattern of rice $H D A C s$ genes indicated that some $H D A C$ genes have different expression profiles. Furthermore, our analysis indicated that expression of HDA705, HDT701, and HDT702 could be affected by salicylic acid, jasmonic acid or abscisic acid. Expression of HDA714, SRT702, and SRT701 could be modulated by abiotic stresses, such as cold, mannitol and salt. These results indicate that different $H D A C$ genes have distinct expression patterns and members of rice HDAC families may be involved in plant response to environmental stresses.
\end{abstract}

(C) 2007 Elsevier Inc. All rights reserved.

Keywords: Histone deacetylase; RPD3/HDA1 family; SIR2 family; HD2 family; Expression; Rice

Acetylation and deacetylation are catalyzed by histone acetyltransferases (HATs) and histone deacetylases (HDACs), respectively. Both enzymes are members of distinct gene families and exist as multiprotein complexes. Many of the recently identified HATs and HDACs turned out to be transcriptional co-activators and co-repressors, thus establishing a direct link between histone acetylation and regulation of gene transcription mechanisms. Acetylation of the histones is often associated with increased gene activity; whereas deacetylation of histones is correlated with transcriptional repression.

Eukaryotic HDACs can be grouped into three major families, namely RPD3/HDA1 superfamily, SIR2 family, and HD2 family, based on their primary homology to the yeast HDACs, RPD3/HDA1, SIR2, and the plant-specific HDAC, HD2 [1]. In Arabidopsis, mutations in a RPD3/

\footnotetext{
* Corresponding authors. Fax: +88622931 8940 (K. Wu), +86 20 37252978 (J. Duan). Duan).

E-mail addresses: kewu01@gmail.com (K.Wu),duanj@scbg.ac.cn (J.
}

$H D A 1$-type $H D A C, H D A 6$, affected transgene expression, DNA methylation, and regulation of rRNA genes [2-4]. Another Arabidopsis RPD3/HDAl-type HDAC, HDA19, is involved in a range of plant development processes and plant response to environmental stresses [5-9]. A recent study indicates that another RPD3/HDAl-type $H D A C$, $H D A 18$, was required for the cellular patterning in the root epidermis in Arabidopsis [10]. These studies demonstrate that members of RPD3/HDA1 HDAC family play essential roles in plant development and plant response to environment.

Previously, Pandey [1] reported sequence analyses of HDAC families from Arabidopsis. In this paper, we analyzed genes encoding RPD3/HDA1, SIR2, and HD2 families of HDACs in rice, a model monocot species. We also investigated the expression profiles of the rice $H D A C$ genes. Our analysis indicates differential expression patterns among members of the $H D A C$ gene families in rice. In addition, some $H D A C$ genes also respond differently to plant hormones and environmental stresses. 


\section{Materials and methods}

Domain predictions. The protein sequences of all HDAC proteins were analyzed for recognizable domains using BLAST-based NCBI conserved domain searches (http://www.ncbi.nlm.nih.gov/Structure/cdd/wrpsb.cgi) [11]. The low-complexity filter was turned off and the expect value was set at one in order to detect short domains or regions of less conservation in this analysis. The $E$ value determined by these searches that indicates the strength of the alignment to the Pfam version 11.0 (pfam01429) or CDD version 2.02 (cdd1396). Domains were also verified using the HMMERbased SMART Web site (http://smart.embl-heidelberg.de) to search both SMART and PFAM domains [12].

Phylogenetic analysis. The complete group of nonreduntant Arabidopsis and rice HDAC proteins was aligned using Clustal X [13]. For all proteins analyzed, the regions of the HDACs used for the alignment began at the conserved HDAC domain. Neighbor joining was then performed with a bootstrap analysis of 1000 replicates using MEGA 2.0 [14].

Plant materials. seven-day-old seedlings of $\mathrm{Pi}-1$ rice were sprayed with $100 \mu \mathrm{M}$ salicylic acid (SA), jasmonic acid (JA) or abscisic acid (ABA) in the closed pots at a growth chamber. Water-spray was used as mock controls. Leaves were collected at $24 \mathrm{~h}$ after treatments. For treatments with abiotic stresses, seven-day-old seedlings of Pi-1 were treated with $0.5 \mathrm{M}$ mannitol, $0.3 \mathrm{M} \mathrm{NaCl}$ or cold $\left(4^{\circ} \mathrm{C}\right)$ for $24 \mathrm{~h}$.

$R N A$ isolation and semi-quantitative RT-PCR analysis. Total RNA was prepared using Trizol reagent (Invitrogen, http://www.invitrogen.com). RNA was treated with the DNase and then used for the synthesis of first strand cDNA with an oligo-dT primer. Gene-specific primers used for PCR were listed in Table 1. Conditions of the PCR were as follows: $94{ }^{\circ} \mathrm{C}$ for $2 \mathrm{~min}, 30$ cycles of $94{ }^{\circ} \mathrm{C}$ for $30 \mathrm{~s}, 56^{\circ} \mathrm{C}$ for $30 \mathrm{~s}, 72^{\circ} \mathrm{C}$ for $2 \mathrm{~min}$, followed by $72{ }^{\circ} \mathrm{C}$ for $10 \mathrm{~min}$ for most $H D A C$ genes. For $H D A 714$ and $H D T 701$, annealing temperature of $53{ }^{\circ} \mathrm{C}$ was used. For RT-PCR analysis, at least three experiments with similar results were performed for each gene. RT-Q-PCR analysis was amended by McGrath et al. [15] and was performed by the Quantity One software.

\section{Results and discussion}

\section{Identification of HDACs encoded by rice genome}

We obtained the sequences of HDACs from the plant chromatin databases (http://www.chromdb.org). Accord- ing to the ChromDB nomenclature, Arabidopsis genes are numbered between 1 and 99, and rice genes are numbered 701-799.

The similarity of the HDACs to current domain models was evaluated by National Center for Biotechnology Information (NCBI) conserved domain searches [16] and HMMER-bases searches using SMART [12]. A total of 14 representatives possessing the complete HDAC domain (Pfam designation PF00850) that defines the RPD3/HDA1 superfamily were identified in rice (Table 2). Iterative TBLASTN searches were performed between these species using all HDAC proteins to find the complete complement of HDAC proteins in the rice genomes. The $E$ value for presence of an HDAC is indicated in Table 2 using two different alignment methods (BLAST and HMMER) to either the PFAM HDAC or the CDD HDAC.

\section{Phylogenetic analysis and identification of putative HDAC motifs of RPD3/HDA1 superfamily HDACs}

The HDACs from the Arabidopsis and rice sequences were aligned using Clustal X [13]. Fig. 1 shows an unrooted phylogenetic tree illustrating the relationships among the Arabidopsis and rice RPD3/HDA1 superfamily proteins, produced by aligning their HDAC domains. The plant proteins were classified into four different classes according to the phylogenetic analysis and consideration of the protein structure and sequence similarity outside of the HDAC (Table 2). Eight Class I proteins of rice can be group into four distinct branches (Fig. 1). Among them, HDA701, HDA707, and HDA709 are grouped into one branch.

\section{Class I motif}

Most mammalian HDACs contain nine conserved domains [17] and a histidine residue (e.g., His ${ }^{141}$ in

Table 1

Primers used for RT-PCR

\begin{tabular}{lll}
\hline Gene name & Forward primer sequence & Reverse primer sequence \\
\hline$H D A 701$ & ACTCCATAGACGGCATCAGG & AAGTAGAGCTTCCGGTGCAG \\
$H D A 702$ & GTTGGGTTGCTTCAACCTGT & GAGCTCATGACCAAGTGCAA \\
$H D A 703$ & ATTACTGCCTGAATGTCCCG & GCAGCGTGCAACATTTCTTA \\
$H D A 704$ & GAGCTGGTGCCAAGAAAGTC & AACTCCCACCTCATTTCTG \\
$H D A 705$ & ATGCTTTAAATGTGCCCCTG & GCAGTCTGCATGACCTTTCA \\
$H D A 707$ & ATGCAAGTTCCTCACCAAGG & AGGAATGAATGCCACAGGAG \\
$H D A 709$ & CCGGAGTACGTCAACCTCAT & ATTGTCGTCGCCGAGTAAAC \\
$H D A 710$ & CAGAACGCCTTCTTGGACTC & CACGTTCACGCTGTAGTGCT \\
$H D A 711$ & ATTACTGCCTGAATGTCCCG & GCAGCGTGCAACATTTCTTA \\
$H D A 712$ & CTATTTCTCGACGCGACTCC & TGCGAGCTCTGTTGGATATG \\
$H D A 713$ & GTGCCAAGAAGCTGTGTGAA & AGTAACCATCAGCACGGTCC \\
$H D A 716$ & TGCTGTACGACGAGAGGATG & CTGAGGAGCCCTTGTTGAAG \\
$H D T 701$ & CTATCCTGGCACCGGTAAGA & GGCTGAAACGAGGATGATGT \\
HDT702 & GTAGACGCACGACGGAAAAT & AGCTTACCCGCCCACTTATT \\
$S R T 701$ & CCCACCTCCCTTTCTTTTC & AGCCTGAGAAAGGTGCAAAA \\
$P R 10 a$ & CTGGGCAATCCTGTGTAGGT & AATGAAACGTGCAACATCCA \\
Actin & TGGCTATGCCGAGAAGCTAT & GATGCACCAGGAACACCTTT \\
\hline
\end{tabular}




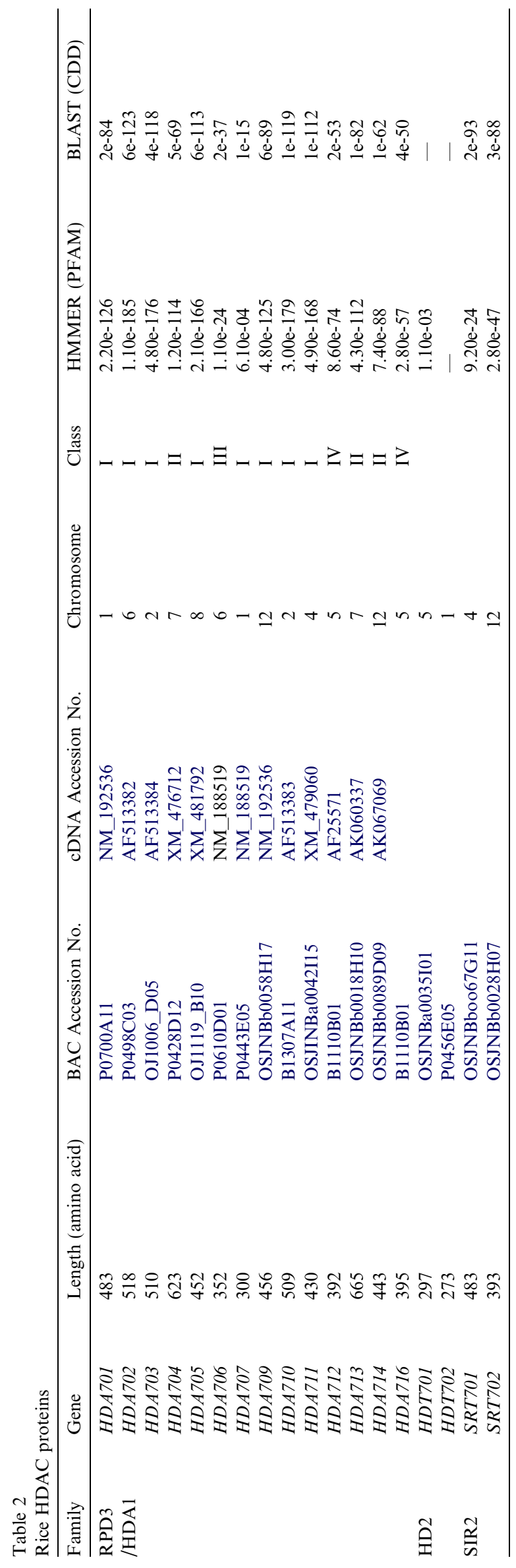

HDAC1), which are essential for catalytic activity [18]. In rice, HDA702, HDA710, and HDA703 (OsHDAC1-3) contains these nine conserved domains and the histidine residue $[17,19]$. In addition to the nine conserved domains and the histidine residue, a conserved cysteine residue was also found in HDA701 (Cys ${ }^{177}$ ), HDA705 $\left(\mathrm{Cys}^{164}\right)$, HDA709 $\left(\right.$ Cys $\left.^{169}\right)$, HDA711 (Cys $\left.{ }^{147}\right)$, except for HDA707 of rice (Fig. 2A). These observations suggest that most Class I RPD3/HDA1 HDAC proteins are functional enzymes in rice and the plant RPD3/HDA1-type Class I domains are actually deacetylase domains.

Several potential post-transcriptional modification sites including a potential $\mathrm{N}$-glycosylation site (NWS) at Asn have been identified in the mammalian [17]. This site is also found in HDA701, HDA703, HDA709, and HDA710 of rice. This site is changed into NWA in HDA702, HDA705, and HDA711, but cannot be found in HDA707.

\section{Class II motif}

The Class II HDACs have been well conserved in higher eukaryotes [20]. There are five clearly conserved sequence motifs, i.e., RPPGHHA, GFCXXN, DøDøHHGXGTQ, $\mathrm{VX}(\mathrm{Y} / \mathrm{F}) \mathrm{SXH}, \varnothing \mathrm{EGGY}$ in the yeast or mammalians, which are limited to the catalytic regions and does not extend to the N-terminal domains ( $\mathrm{X}$ represents any amino acid while $\varnothing$ indicates a hydrophobic residue) [20]. The five conserved domains, the histidine residue and the cysteine residue were also found in HDA704 ( $\mathrm{His}^{331}$, $\mathrm{Cys}^{341}$ ), HDA713 (His ${ }^{144}, \mathrm{Cys}^{154}$ ), and HDA714 (His ${ }^{222}$, $\mathrm{Cys}^{232}$ ) (Fig. 2B), suggesting that Class II HDAC proteins are also functional enzymes in rice. This evolutionary conservation suggests that these enzymes may perform critical functions [20].

\section{Class III motif}

Only rice HDA706 belongs to Class III HDAC members. The nine conserved domains, the histidine (His ${ }^{164}$ ) and the cysteine $\left(\mathrm{Cys}^{174}\right)$ residues were also found in HDA706 (Fig. 2C).

\section{Class IV motifs}

Rice HDA712 and HDA716 belong to the Class IV HDAC members. Only seven conserved domains, the histidine residue and the cysteine residue were also found in HDA712 ( $\mathrm{His}^{161}, \mathrm{Cys}^{171}$ ) and HDA716 ( $\mathrm{His}^{164}, \mathrm{Cys}^{174}$ ) (Fig. 2D).

\section{Domain architecture of RPD3/HDA1 superfamily HDACs from rice}

\section{Class I HDACs}

The HDAC domains are located within 300 amino acids of the $\mathrm{N}$-terminus in all class I HDAC domain proteins (Fig. 3). The C-terminal regions of the Class I proteins, HDA701, HDA702, HDA705, HDA707, HDA709, and HDA710, contain a low complexity. In addition, the N-terminal regions of HDA702, HDA703, and HDA709 also have a low complexity domain. HDA701 and HDA707 


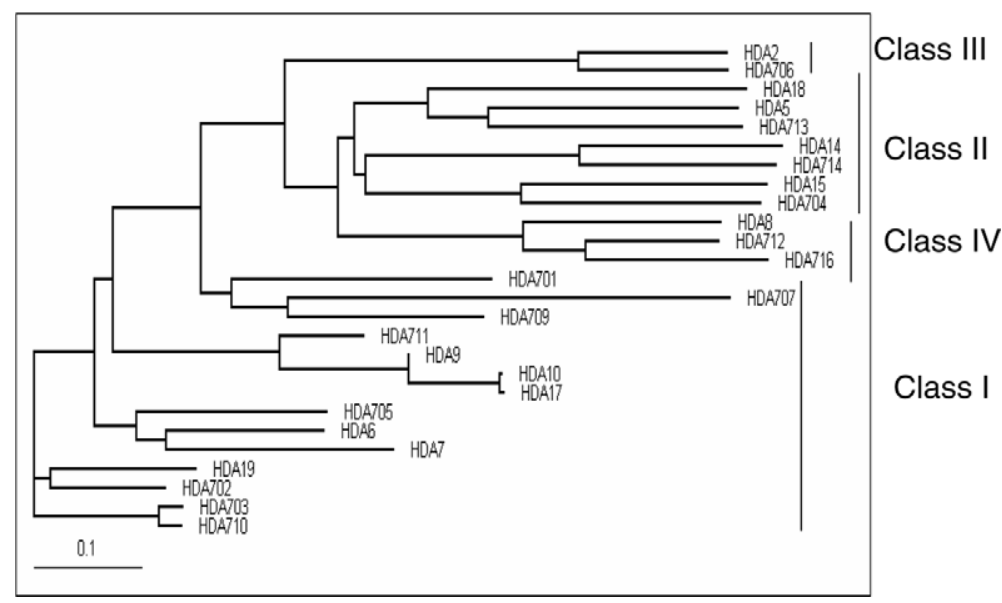

Fig. 1. Phylogenetic analysis of the members of RPD3/HDA1-type HDACs in Arabidopsis and rice. Arabidopsis proteins are numbered between 1 and 19, and rice proteins are numbered 701-716.

occur immediately adjacent to each other on chromosome I, suggesting a gene duplication event. Similarly, HDA703 and HDA710 also occur immediately adjacent to each other on chromosome II, suggesting another gene duplication event.

\section{Class II HDACs}

The rice genome possesses three Class II proteins, which are designated to HDA704, HDA713, and HDA714. All of them have a complete HDAC domain. HDA704 contains a RanBP zinc-finger domain that has been implicated in nucleocytoplasmic transport and nuclear envelope localization $[1,21]$. HDA704 also contains two low complexity regions-a Glu/Asp-rich region that forms a predicted amphpathic $\alpha$-helical structure, and an Ala/Leu-rich region of hydrophobic $\alpha$-helices in its $\mathrm{N}$-terminal region. The $\mathrm{C}$ terminal and $\mathrm{N}$-terminal regions of Class II protein HDA713 both contain low complexity regions-an Alarich region that is essential for repression and also interacts with TBP (TATA-box binding protein), and a Ser-rich region that plays a crucial role in splicing and is implicated in splice site selection in metazoa [22].

\section{Class III HDACs}

A new class of HDAC proteins, designated Class III, is represented in rice by HDA706. HDA706 has a complete HDAC domain and a low complexity region-an Ala/ Ser/Pro-rich region. The Pro-rich region is deeply involved in transcriptional regulation [23].

\section{Class IV HDACs}

The rice genome encodes two additional HDAC proteins in the RPD3/HDA1 superfamily, respectively, namely HDA712 and HDA716. Both of them contain a complete HDAC domain. HDA712 has a low complexity-Ser/ Ala-rich region in its $\mathrm{N}$-terminal region (Fig. 3). HDA712 and HDA716 in rice occur immediately adjacent to each other on chromosome $\mathrm{V}$, suggesting a gene duplication event.

\section{Analysis of the rice HDAC expression}

To investigate the expression of rice $H D A C$ genes, the mRNA accumulation patterns were examined for all of the rice $H D A C$ genes by RT-PCR. As shown in Fig. 4A, HDA702, HDA704, HDA712, HDT701, and SRT701 mRNAs were detected in leaves, stems, and roots; however, the levels of accumulation differed among different organs. HDA706 and SRT702 mRNAs were detected in leaves and stems but not in roots. In comparison, HDA705 and HDT702 mRNAs were only detected in roots. The transcripts of HDA701, HDA703, HDA707, HDA709, $H D A 711, H D A 713$, and $H D A 716$ were not detected (data not shown). The differences in expression patterns may reflect specific functions of different HDACs.

\section{Response of HDAC genes to $S A, J A$, and $A B A$}

SA and JA mediate plant defense response, whereas ABA is the major plant hormone in water stress signaling and regulates plant water balance and osmotic stress tolerance [24]. In Arabidopsis, it has been demonstrated that the expression of $H D A 6$ and $H D A 19$ can be induced by JA [8], whereas the expression of $A t H D 2 C$ (HDT3) was repressed by ABA [25]. RT-PCR was conducted using total RNAs isolated from leaves of seven-day-old rice seedling subjected to SA, JA, and ABA treatment. PRIOa gene was selected as a control since previous study has shown that it can be induced by JA and SA [26]. As shown in Fig. 4B, PR10a gene can be induced by JA, SA, and ABA. In the RPD3/HDAl family, the expression of HDA705, which has a high sequence homology with Arabidopsis HDA6 (67\%) and HDA19 (58\%), was induced by JA. In addition, the expression of $H D A 702$ and $H D A 704$ was induced by SA, JA, and ABA. In HD2 family members, the expression of $H D T 701$, which has a high sequence 


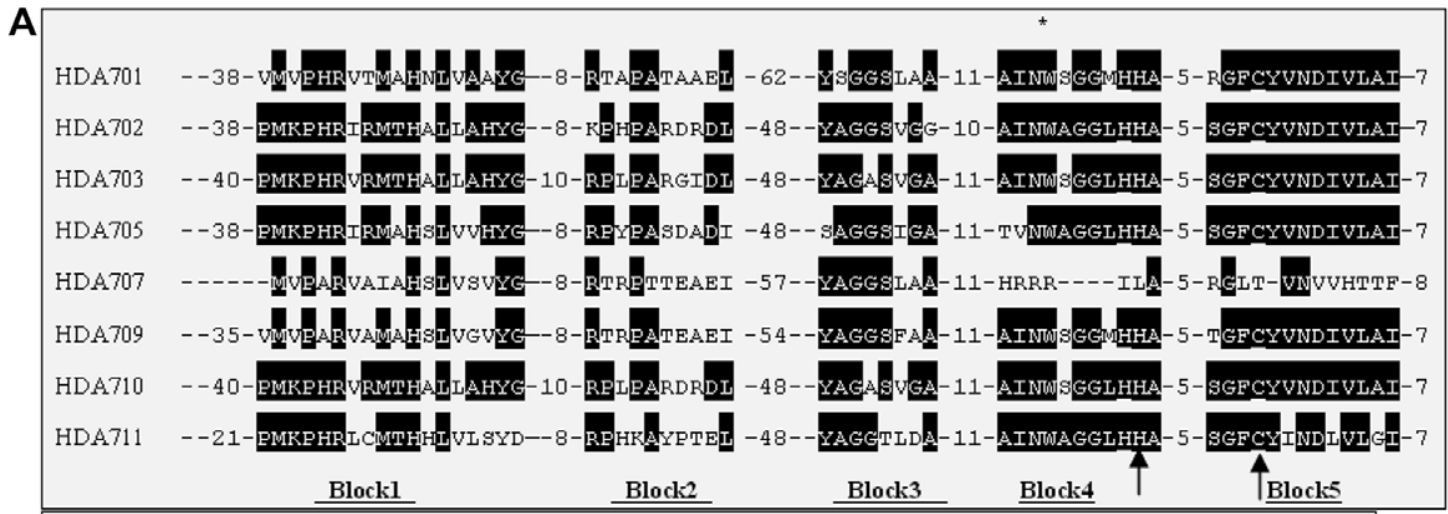

HDA701 BULYYDIDVHHG-29-DVGKRGPGRYCALNVP-28-AVVLOCGADSIAGDRLGEINLT-13-ENLPLLLLGGGGY-152

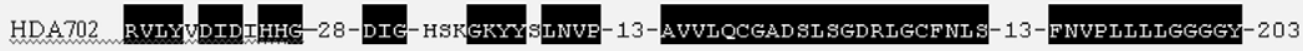
HDA703 BULYIDID IHHE-28-DIG-YSEGKYYCLNUP-13-AVULQCGADSISGDRLGCFNLS-13-FNVDLLLIGGGGY-190 HDA705 RULYVDIDVHHE-28-DVG-ACEGRHUALLVE-13-VUVLOCGADSIAEDRLGCFNLS-13-ENIBMMVLGGGGY-136

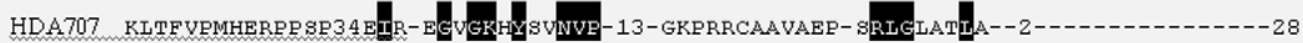

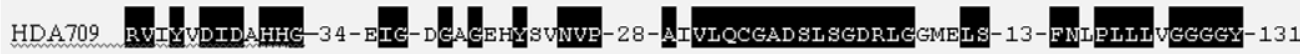
HDA710 BVLYIDIDIHHG-28-DIG-YSEGKYYCLNVE-27-AVVLQCGADSL SGDRLGCFNLS-13-ENVDLLLIGGGGY-189 HDA711 RULYIDIDVHHE-29-DIG-EREGKYYAINIE-13-AIVLOCGADSIARDRLGCFNLS-13-ENIELIVIGGGGY-130 Block6 Block7 Block8 Block9

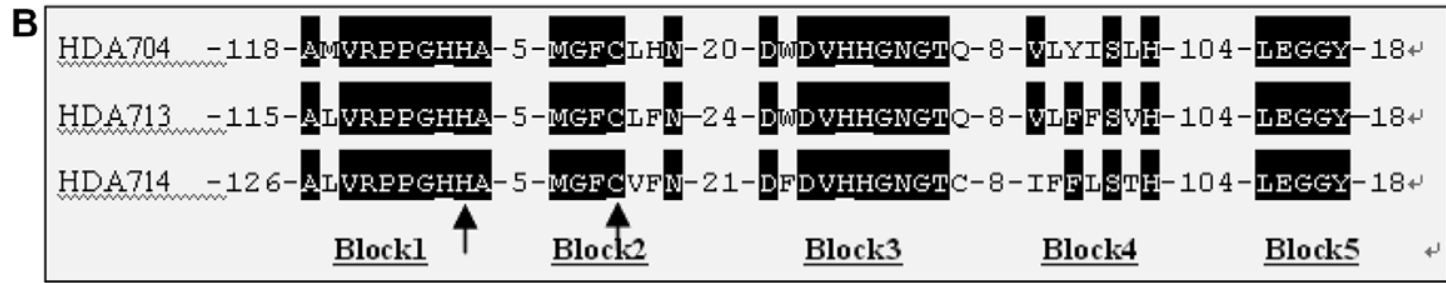

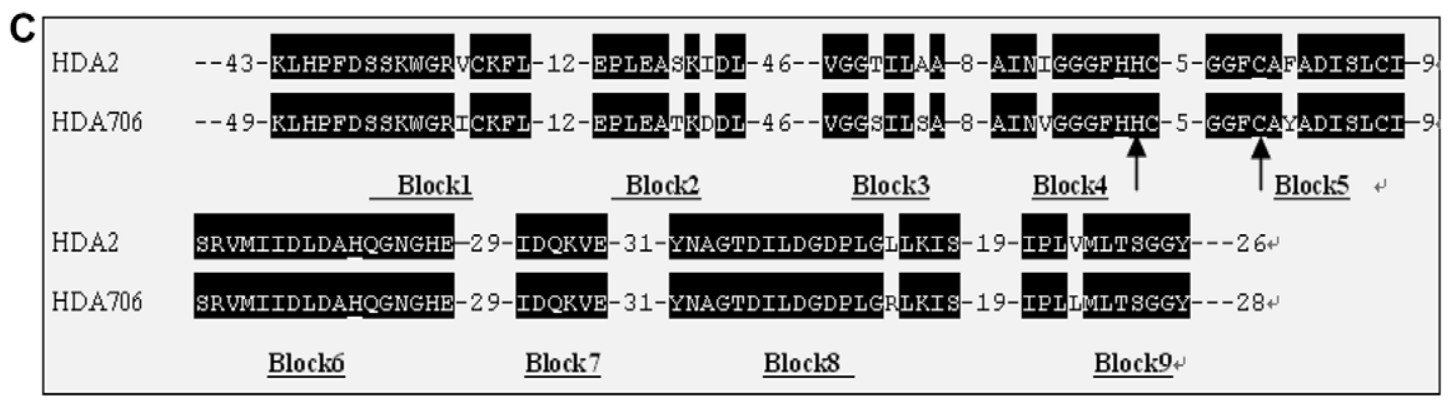

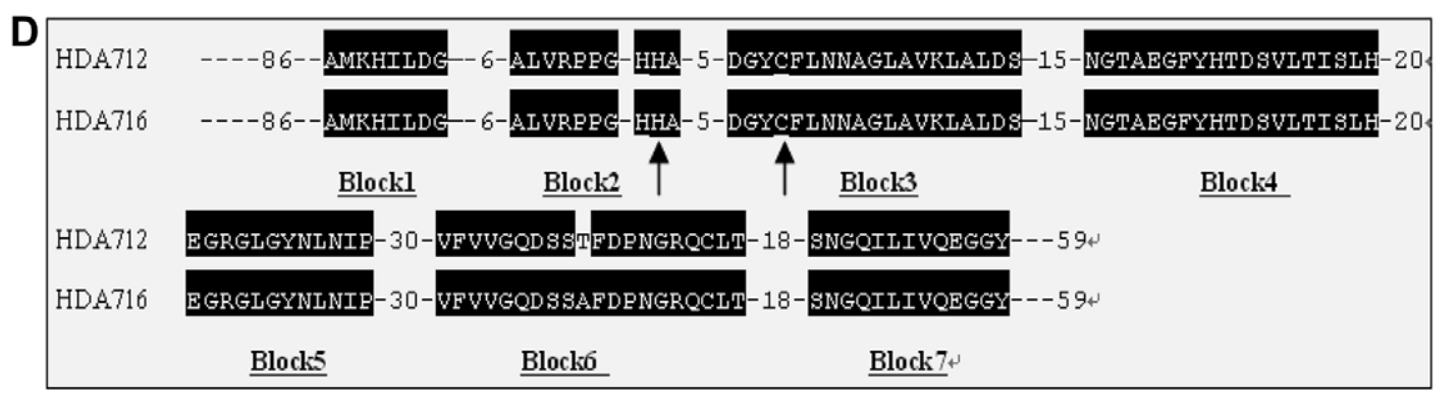

Fig. 2. Alignment of the blocks of RPD3/HDA1-type HDACs from rice or Arabidopsis. Amino acid sequences for Class I HDACs from rice were aligned using the DNAMAN program. Identical amino acids are boxed in white letters on a black background. Arrows indicate amino acids identified by mutagenesis to play an important catalytic and/or structural role in human HDAC1 [18]. (A) Alignment of the blocks of Class I HDAC from rice. A potential $N$-glycosylation site (NWS) at Asn in the mammalian HDACs [17] was also found in rice HDACs and was indicated by an asterisk. (B) Alignment of the blocks of Class II HDAC from rice. (C) Alignment of the blocks of Class III HDAC from rice and Arabidopsis. (D) Alignment of the blocks of Class IV HDAC from rice. 


\section{Class I HDACs}

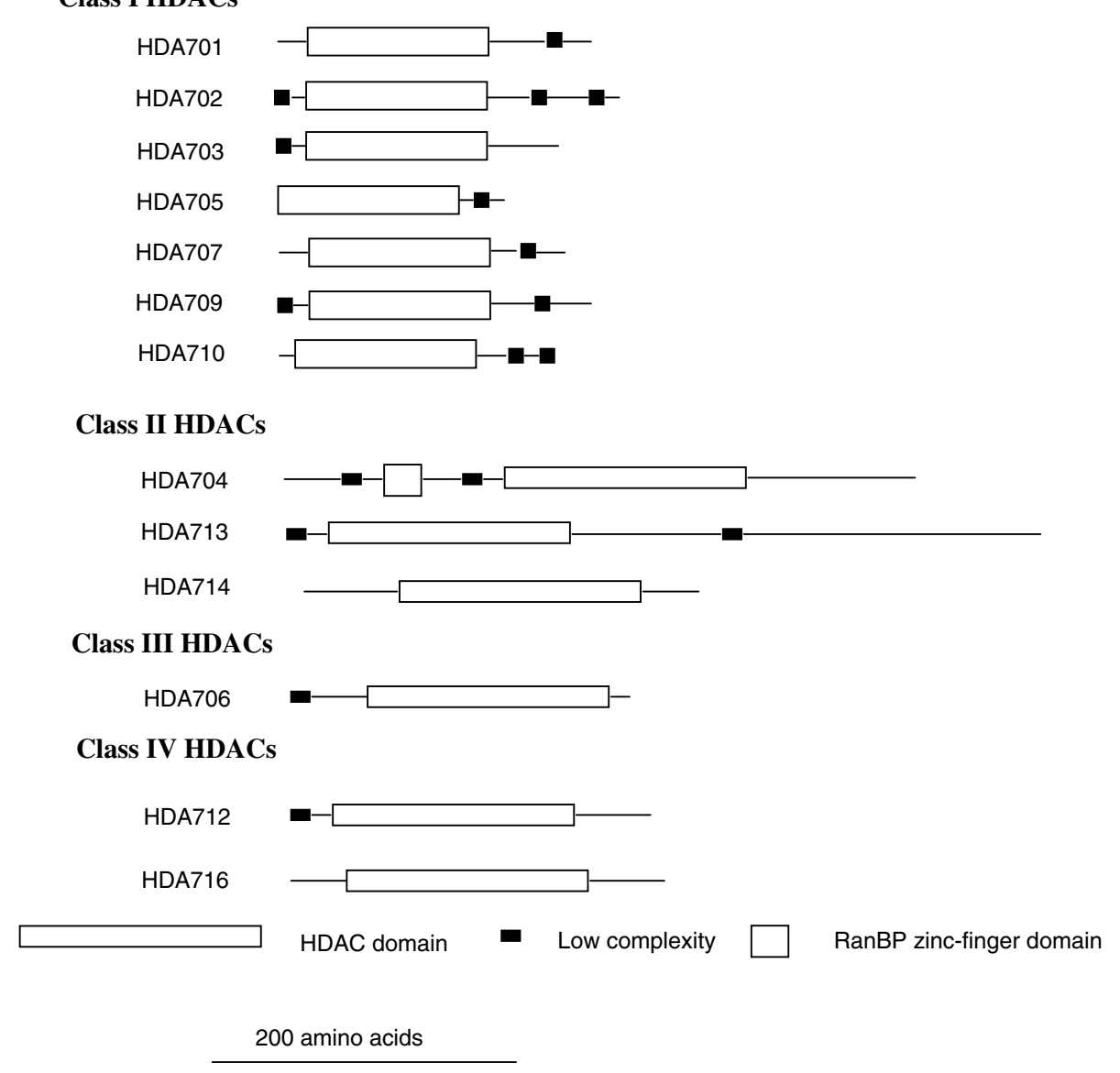

Fig. 3. Domain organization of the RPD3/HDA1-type HDACs proteins in rice. The locations and size of domains are shown by the use of different shape as indicated. The proteins belonging to each class are grouped together.

homology with Arabidopsis HDT1 (43\%) and HDT3 (41\%), was also induced by JA. In addition, the mRNA accumulation of HDT702, another HD2 family member, was induced by both SA and JA. In SIR2 family members, the expression of $S R T 701$ was induced by JA but decreased by SA and ABA, whereas the expression of $S R T 702$ was decreased by SA, JA, and ABA.

\section{Response of HDAC genes to abiotic stress treatments}

To investigate the expression pattern of $H D A C$ genes in response to environmental stress conditions, RT-PCR was conducted using total RNA isolated from leaves of sevenday-old rice seedlings that were subjected to cold $\left(4^{\circ} \mathrm{C}\right)$, mannitol and salt $(\mathrm{NaCl})$ treatment. After cold treatment, the expression of HDA704, HDA712, SRT701, SRT702, and PR10a was decreased, while the expression of HDA702, HDA706, HDA714, and HDT701 was increased (Fig. 4C). After mannitol treatment, the expression of HDA704, HDA706, HDA712, HDT701, SRT701, $S R T 702$, and PR10a was decreased, while the expression of $H D A 702$ was increased. After $\mathrm{NaCl}$ treatment, the expression of $H D A 702$ was increased, whereas the expression of HDA704, HDA706, HDA712, HDA714, HDT701, $S R T 701, S R T 702$, and PR10a was decreased. These results suggest that the expression of members of $H D A C$ gene families can be modulated by abiotic stresses and some of them may be involved in the plant response to abiotic stresses.

Comparison of transcript profiles in various rice tissues by RT-PCR analysis indicates that members of the $H D A C$ genes are expressed at different levels. Previously, it was found that the rice RPD3/HDA1-type $H D A C, H D A 702$, the maize ZmRPD3/HD1B-I, the Arabidopsis HDA19, and the Arabidopsis HD2-type HDACs, AtHD2A, $A t H D 2 B$, and $A t H D 2 C$ were all constitutively expressed [19,27-30]. Similarly, we found that rice $H D A 702$, HDA704, HDA712, HDT701, and SRT701 were expressed in all the tissues tested, suggesting that these genes may be necessary for the plant growth and development. In comparison, HDA705, HDA706, HDA714, HDT702, and $S R T 702$ were expressed in specific tissues only, suggesting that some rice $H D A C$ genes may have adopted different functions.

The plant hormones ABA, JA, SA and ethylene are involved in regulating defense gene expression during adaptive responses to abiotic and biotic stresses in plants [31-33]. Recent studies suggested that HDACs and histone acetylation may play a key role in the integration of ethyl- 

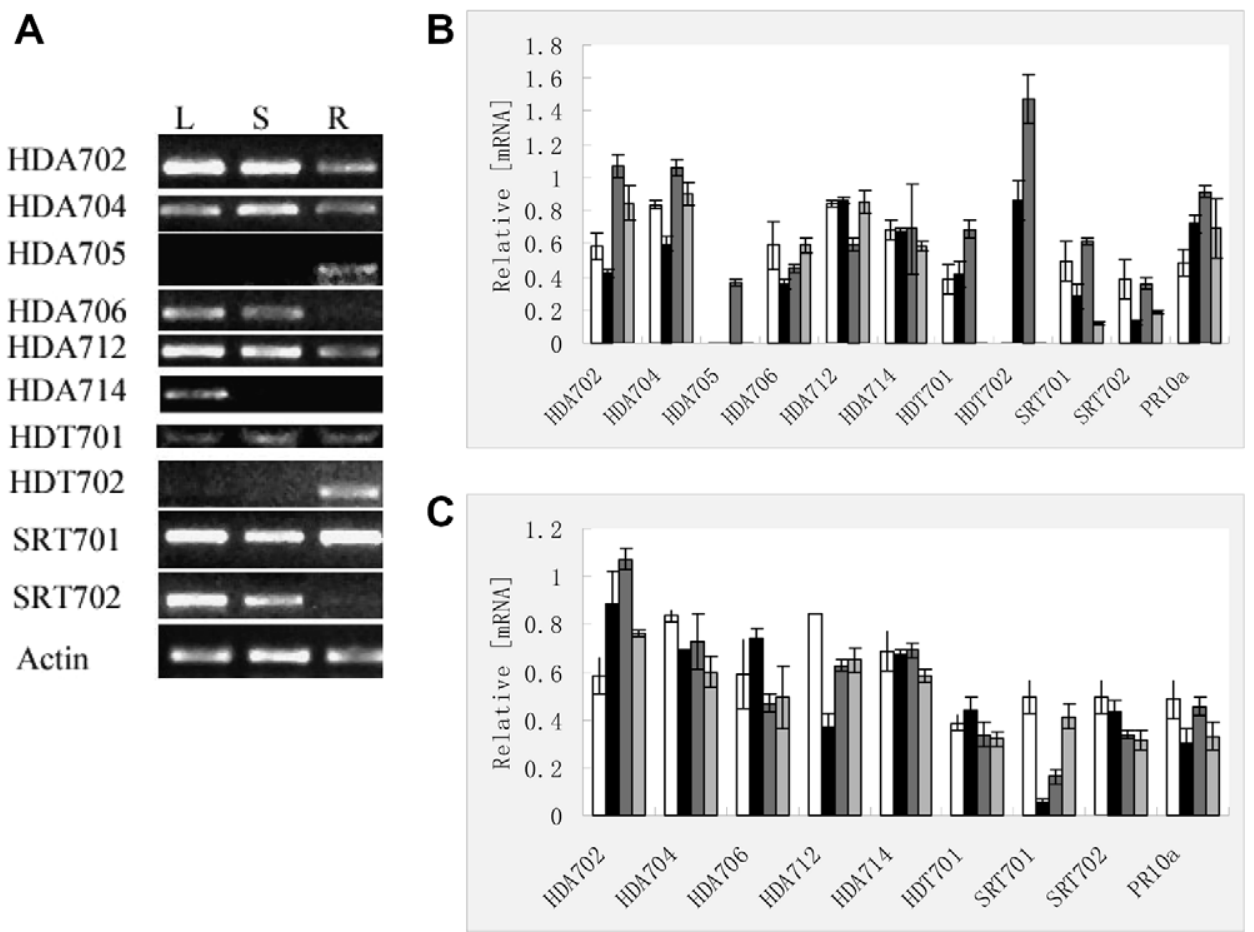

Fig. 4. RT-PCR analysis of RPD3/HDA1-type HDAC expression in tissue and treatment with plant hormone and abiotic stresses. (A) Total RNAs were isolated from the leaves (L), stems (S), and roots (R) of 7-day-old seedlings. The actin was used as a control. (B) Total RNAs were isolated from rice 7-dayold seedling leaves treated without hormone (CK, white bars), or with $100 \mu \mathrm{M} \mathrm{SA}$ (black bars), JA (dark gray bars) and ABA (gray bars) for $24 \mathrm{~h}$. (C) Total RNAs were isolated from rice 7-day-old seedling leaves treated with no hormone (CK, white bars), cold ( $\left.4{ }^{\circ} \mathrm{C}\right)$ (black bars), $0.5 \mathrm{M}$ mannitol (dark gray bars), $0.3 \mathrm{M} \mathrm{NaCl}$ (gray bars) for $24 \mathrm{~h}$. RT-PCR amplified as outlined in Materials and methods, using gene-specific primers. The actin and PR10a were used as controls. Average data with standard errors from three independent biological replicates are shown.

ene, JA and ABA signals for the regulation of stress response genes [25]. We found that different rice $H D A C$ genes responded differently to environmental stresses and to hormones, SA, JA, and ABA. For examples, JA induced the accumulation of HDA705 and HDT701 as well as PR10a transcripts. SA and JA induced the expression of HDT702. On the other hand, ABA repressed the expression of HDT701, HDT702, SRT701, and SRT702. These observations suggested that some $H D A C$ genes may play a role in the plant defense response.

More recent studies suggested that histone acetylation and deacetylation are involved in plant response to abiotic stresses [25,34-37]. Our results indicate that cold stress decreased the expression of HDA712, SRT702, and $S R T 701$. Mannitol induced the expression of HDT701, but repressed the expression of $S R T 701$ and $S R T 702$. High salt stress induced the expression of HDT701 and HDA714, whereas decreased $S R T 701$ and SRT702. These observations suggested that members of $H D T$ family and SIR 2 family may be involved in the abiotic stress signal pathways.

\section{Acknowledgment}

This work was supported by grants from the Natural National Science Foundation of China (No. 30471002) and the Natural Science Foundation of Guangdong Province, China (No. 05103637).

\section{References}

[1] R. Pandey, A. Muller, C.A. Napoli, et al., Analysis of histone acetyltransferase and histone deacetylase families of Arabidopsis thaliana suggests functional diversification of chromatin modification among multicellular eukaryotes, Nucleic Acids Res. 30 (2002) 50365055.

[2] J. Murfett, X. Wang, G. Hagen, et al., Identification of Arabidopsis histone deacetylase HDA6 mutants that affect transgene expression, Plant Cell 13 (2001) 1047-1061.

[3] W. Aufsatz, W.F. Mette, J. van der Winden, et al., HDA6, a putative histone deacetylase needed to enhance DNA methylation induced by double-stranded RNA, EMBO J. 21 (2002) 6832-6841.

[4] A.V. Probst, M. Fagard, F. Proux, et al., Arabidopsis histone deacetylase $H D A 6$ is required for maintenance of transcriptional gene silencing and determines nuclear organization of rDNA repeats, Plant Cell 16 (2004) 1021-1034.

[5] K. Wu, K. Malik, L. Tian, et al., Functional analysis of a RPD3 histone deacetylase homolog in Arabidopsis thaliana, Plant Mol. Biol. 44 (2000) 167-176.

[6] L. Tian, Z.J. Chen, Blocking histone deacetylation in Arabidopsis induces pleiotropic effects on plant gene regulation and development, Proc. Natl. Acad. Sci. USA 98 (2001) 200-205.

[7] L. Tian, J. Wang, M.P. Fong, et al., Genetic control of developmental changes induced by disruption of Arabidopsis histone deacetylase 1 (AtHD1) expression, Genetics 165 (2003) 399-409.

[8] C. Zhou, L. Zhang, J. Duan, et al., HISTONE DEACETYLASE19 is involved in jasmonic acid and ethylene signaling of pathogen response in Arabidopsis, Plant Cell 17 (2005) 1196-1204.

[9] J.A. Long, C. Ohno, Z.R. Smith, et al., Topless regulates apical embryonic fate in Arabidopsis, Science 312 (2006) 1520 1523. 
[10] C. Xu, C. Liu, Y. Wang, et al., Histone acetylation affects expression of cellular patterning genes in the Arabiodopsis root epidermis, Pro. Natl. Acad. Sci. USA 102 (2005) 14469-14474.

[11] A. Marchler-Bauer, J.B. Anderson, C. DeWeese-Scott, et al., CDD: a curated Entrez database of conserved domain alignments, Nucleic Acids Res. 31 (2003) 383-387.

[12] J. Schultz, R.R. Copley, T. Doerks, et al., SMART: a web-based tool for the study of genetically mobile domains, Nucleic Acids Res. 28 (2000) 231-234.

[13] J.D. Thompson, T.J. Gibson, F. Plewniak, et al., The CLUSTAL X windows interface: flexible strategies for multiple sequence alignment aided by quality analysis tools, Nucleic Acids Res. 25 (1997) 48764882.

[14] S. Kumar, K. Tamura, I.B. Jakobsen, et al., MEGA2: molecular evolutionary genetics analysis software, Bioinformatics 17 (2001) $1244-1245$.

[15] K.C. McGrath, B. Dombrecht, J.M. Manners, et al., Repressor- and activator-type ethylene response factors functioning in jasmonate signaling and disease resistance identified via a genome-wide screen of Arabidopsis transcription factor gene expression, Plant Physiol. 139 (2005) 949-959.

[16] S.F. Altschul, T.L. Madden, A.A. Schäffer, et al., Gapped BLAST and PSI-BLAST: a new generation of protein database search programs, Nucleic Acids Res. 25 (1997) 3389-3402.

[17] E. Hu, Z. Chen, T. Fredrickson, et al., Cloning and characterization of a novel human class I histone deacetylase that functions as a transcription repressor, J. Biol. Chem. 275 (2000) 15254 15264 .

[18] C.A. Hassig, J.K. Tong, T.C. Fleischer, et al., A role for histone deacetylase activity in HDAC1-mediated transcriptional repression, Proc. Natl. Acad. Sci. USA 95 (1998) 3519-3524.

[19] I.C. Jang, Y.M. Pahk, S.I. Song, et al., Structure and expression of the rice class-I type histone deacetylase gene OsHDAC1-3: OsHDACI overexpression in transgenic plants leads to increased growth rate and altered architecture, Plant J. 33 (2003) 531-541.

[20] C.M. Grozinger, C.A. Hassig, S.L. Schreiber, Three proteins define a class of human histone deacetylases related to yeast Hda1p, Proc. Natl. Acad. Sci. USA 96 (1999) 4868-4873.

[21] I.R. Vetter, C. Nowak, T. Nishimoto, et al., Structure of a Ranbinding domain complexed with Ran bound to a GTP analogue: implication for nuclear transport, Nature 398 (1999) 39-46.

[22] S. Lopato, C. Forstner, M. Kalyna, et al., Network of interactions of a novel plant-specific Arg/Ser-rich protein, atRSZ33, with atSC35like splicing factors, J. Biol. Chem. 277 (2002) 39989-39998.
[23] H. Tamai, M. Iwabuchi, T. Meshi, Arabidopsis GARP transcriptional activators interact with the pro-rich activation domain shared by G-box-binding bZIP factors, Plant Cell Physiol. 43 (2002) 99-107.

[24] L. Xiong, K.S. Schumaker, J.K. Zhu, Cell signaling during cold, drought, and salt stress, Plant Cell 14 (2002) S165-S183.

[25] S. Sridha, K. Wu, Identification of $A t H D 2 C$ as a novel regulator of abscisic acid responses in Arabidopsis, Plant J. 46 (2006) 124-133.

[26] N. Midoh, M. Iwata, Cloning and characterization of a probenazoleinducible gene for an intracellular pathogenesis-related protein in rice, Plant Cell Physiol. 37 (1996) 9-18.

[27] K. Wu, L. Tian, K. Malik, et al., Functional analysis of HD2 histone deacetylase homologues in Arabidopsis thaliana, Plant J. 22 (2000) 1927.

[28] C. Zhou, H. Labbe, S. Sridha, et al., Expression and function of HD2-type histone deacetylases in Arabidopsis development, Plant J. 38 (2004) 715-724.

[29] T. Lechner, A. Lusser, A. Pipal, et al., RPD3-type histone deacetylases in maize embryos, Biochemistry 39 (2000) 1683-1692.

[30] V. Rossi, H. Hartings, M. Motto, Identification and characterization of anRPD3 homologue from maize (Zea mays L.) that is able to complement an rpd3 null mutant of Saccharomyces cerevisiae, Mol. Gen. Genet. 258 (1998) 288-296.

[31] R.R. Finkelstein, S.S. Gampala, C.D. Rock, Abscisic acid signaling in seeds and seedlings, Plant Cell 14 (2002) S15-S45.

[32] K.L. Wang, H. Li, J.R. Ecker, Ethylene biosynthesis and signaling networks, Plant Cell 14 (2002) S131-S151.

[33] J.P. Anderson, E. Badruzsaufari, P.M. Schenk, et al., Antagonistic interaction between abscisic acid and jasmonate-ethylene signaling pathways modulates defense gene expression and disease resistance in Arabidopsis, Plant Cell 16 (2004) 3460-3479.

[34] H.J. Kim, Y. Hyun, J.Y. Park, A genetic link between cold responses and flowering time through FVE in Arabidopsis thaliana, Nat. Genet. 36 (2004) 167-171.

[35] B. Lee, D.A. Henderson, J.K. Zhu, The Arabidopsis cold-responsive transcriptome and its regulation by ICE1, Plant Cell 17 (2005) 31553175.

[36] E.J. Stockinger, Y. Mao, M.K. Regier, et al., Transcriptional adaptor and histone acetyltransferase proteins in Arabidopsis and their interactions with CBF1, a transcriptional activator involved in coldregulated gene expression, Nucleic Acids Res. 29 (2001) 1524-1533.

[37] K.E. Vlachonasios, M.F. Thomashow, S.J. Triezenberg, Disruption mutations of $A D A 2 b$ and GCN5 transcriptional adaptor genes dramatically affect Arabidopsis growth, development, and gene expression, Plant Cell 15 (2003) 626-638. 